\title{
Local therapy in patients with metastatic prostate cancer: a new standard of care?
}

\author{
Benjamin Harper ${ }^{1,2}$, Zachary Klaassen ${ }^{2,3}$, Christopher J. D. Wallis ${ }^{4}$ \\ ${ }^{1}$ Medical College of Georgia, Augusta University/University of Georgia Medical Partnership, Athens, GA, USA; ${ }^{2}$ Division of Urology, Medical \\ College of Georgia at Augusta University, Augusta, GA, USA; ${ }^{3}$ Georgia Cancer Center, Augusta, GA, USA; ${ }^{4}$ Division of Urology, Department of \\ Surgery, University of Toronto, Toronto, ON, USA \\ Correspondence to: Christopher J. D. Wallis, MD, PhD. Division of Urology, Department of Surgery, University of Toronto, 149 College Street, \\ Room 503G, Toronto, ON M5T 1P5, Canada. Email: wallis.cjd@gmail.com. \\ Comment on: Burdett S, Boevé LM, Ingleby FC, et al. Prostate Radiotherapy for Metastatic Hormone-sensitive Prostate Cancer: A STOPCAP \\ Systematic Review and Meta-analysis. Eur Urol 2019;76:115-24.
}

Submitted May 18, 2019. Accepted for publication May 23, 2019.

doi: $10.21037 /$ tcr.2019.05.27

View this article at: http://dx.doi.org/10.21037/tcr.2019.05.27

Historically, the standard of care for patients with metastatic prostate cancer was androgen deprivation therapy (ADT). With the publication of CHAARTED, LATITUDE, and STAMPEDE, there are randomized data (1-3), and metaanalysis of randomized data (4), to support the addition of docetaxel and abiraterone in these patients. While local treatment such as radical prostatectomy and prostate irradiation are standard for patients with localized disease, the role of local therapy in patients with distant metastases has been controversial until very recently (5). Although there is data to suggest that patients with oligometastatic prostate cancer may derive oncologic benefit from prostate directed treatment, many of the published studies to date are retrospective (6). As such, selection bias (with patients with lower overall disease burden being treated more aggressively at the local site and ultimately having a greater survival) may account for many of the observed benefits in these studies.

To date, there are two randomized trials assessing the role of radiotherapy to the prostate in patients with metastatic prostate cancer. In the multi-arm STAMPEDE trial, 2,061 patients with newly diagnosed metastatic prostate cancer were randomized to either a standard treatment control group receiving ADT (an in some cases docetaxel), or to a radiotherapy group that received external beam therapy in addition to standard therapy between 2013 and 2016 (3). Overall there was no significant survival difference between the two groups. However, there was noted an increase in overall and failure-free survival among patients who had a low metastatic burden who received radiotherapy. Similarly, the HORRAD trial randomized 432 patients with PSA $>20 \mathrm{ng} / \mathrm{mL}$ and confirmed bone metastases to either an $\mathrm{ADT}$ alone or ADT + EBRT (7). This analysis showed an improvement in time to PSA progression among patients receiving radiotherapy, but there was no difference in overall survival among the two groups.

The STOPCAP M1 Radiotherapy team used a prospective framework to identify relevant trials assessing the role of primary prostate radiotherapy in patients with metastatic hormone sensitive prostate cancer (8). Identifying three relevant trials, the authors performed meta-analysis of two prospective, randomized controlled trials (HORRAD and STAMPEDE) which have reported analyses of the use of prostate directed radiation therapy + ADT versus ADT alone. The PEACE-1 trial, which has closed for accrual but not yet read out, is planned for inclusion in future metaanalyses on this topic but, to date, provides no relevant data.

Before examining the effect of prostate radiotherapy, it is important to understand the characteristics of the included patients. Among 2,126 patients included in this analysis, all patients had de novo metastatic prostate cancer and the majority had Gleason score $\geq 8$, performance status 0 , and median age was in the late sixties. The vast majority of included men had bone metastasis $(100 \%$ in the HORRAD trial and $89 \%$ in the STAMPEDE report), with rare visceral metastasis. All men received long-term ADT. Prostate radiotherapy regimens differed between the two trials: men in STAMPEDE received either 55 Gy in 20 fractions over 
4 weeks or 36 Gy in six fractions over 6 weeks, while those in HORRAD received 70 Gy in 35 fractions over 7 weeks or an alternate schedule of 57.76 Gy in 19 fractions over 6 weeks. Median time to follow up was 41.9-47 months.

Among all included patients, the authors found no significant difference in overall survival when radiotherapy was given in addition to ADT. Progression free survival (time to symptomatic or radiographic progression, or death) also did not show a significant benefit to receiving radiation along with ADT. There was a statistically significant improvement of biochemical progression, showing at 3 years an absolute improvement of $11 \%$ (36\% of men with $\mathrm{ADT}$ along versus $25 \%$ of men with $\mathrm{ADT}+$ radiotherapy).

However, in keeping with the STAMPEDE report, Burdett et al. noted the survival of patients who received radiation therapy was dependent on the number of bone metastases (interaction HR for overall survival 1.47, 95\% CI, 1.11-1.94) (8). Using a pre-specified criterion, patients were sub-stratified for analysis into $<5$ bone metastases versus $\geq 5$ bone lesions. Patients with fewer than 5 bone metastases had a statistically significant increase in survival when they received radiotherapy in conjunction to $\mathrm{ADT}$ : an absolute improvement of $7 \%$ ( $77 \%$ versus $70 \%, 95 \%$ CI, $2-11 \%)$, and they noted a similar improvement of progression free survival. This shows that patients who have a low metastatic burden may have some benefit to receiving radiation therapy at the time of diagnosis.

While there is a reasonable biologic rationale for local therapy in patients with low-volume metastatic hormone sensitive prostate cancer, before accepting this approach as standard of care, limitations to these data must be highlighted. First, the standard of care has changed since these trials accrued. In the present meta-analysis, no patients received abiraterone and few received docetaxel at the time of diagnosis. These agents are now widely used in this indication and each has a survival benefit whose magnitude exceeds the benefits seen here. The role of radiotherapy in patients receiving these life-prolonging systemic therapies remains to be assessed in the PEACE-1 trial (comparison $\mathrm{B} 1: \mathrm{RT}+$ abiraterone + ADT vs. abiraterone + ADT; comparison $\mathrm{B} 2: \mathrm{RT}+$ docetaxel + $\mathrm{ADT} v$ s. docetaxel + $\mathrm{ADT}$; comparison $\mathrm{B} 3$ : $\mathrm{RT}+$ abiraterone + docetaxel + ADT $v s$. abiraterone + docetaxel $+\mathrm{ADT})$. Further, an ongoing analysis of the STAMPEDE multi-arm trial (arm H vs. A) will compare $\mathrm{RT}+$ docetaxel $+\mathrm{ADT} v s$. docetaxel + ADT.

The authors also point out how changes in imaging modalities, specifically with use of tests with higher sensitivity, may change the definition of metastasis moving forward.
Finally, there remains significant interest in radiotherapy to metastatic sites in patients with oligometastatic prostate cancer. This question is not addressed in the present manuscript but is being assessed in ongoing clinical trials.

Beyond considerations which may affect the validity of the role of radiotherapy in patients with metastatic hormone sensitive disease, there must be careful consideration of the potential toxicity of this approach, especially among patients with uncurable disease for which the estimated survival benefit is 7 months. The STAMPEDE trial assessed toxicity using the Radiation Therapy Oncology Group scale and noted 5\% of patients reported grade 3-4 bladder toxicity, and only $1 \%$ of patients reported grade 3-4 bowel toxicity (3). Minor side effects were more common as patients reported $63 \%$ grade 1-2 bladder toxic effects, and 54\% bowel effects.

Burdett $e t a l$. show strong evidence of a survival benefit with the use of prostate radiotherapy among men with low-burden metastatic, hormone sensitive prostate cancer. As with any invasive treatments planned on a patient, this decision to proceed with treatment requires a strong understanding of both the clinician and the patient as to what the expected benefits are and what the potential side effects are. Moving forward, the completion of the PEACE-1 trial along with the partial data from the STAMPEDE trial may help to show the effect of radiotherapy among patients receiving docetaxel or abiraterone. Other trials, including SWOG 1802, TRoMbone, and STAMPEDE arm M (pending funding approval), are similarly investigating the use of radical prostatectomy as an alternative in patients who are metastatic at presentation.

\section{Acknowledgments}

Funding: None.

\section{Footnote}

Provenance and Peer Review: This article is commissioned and reviewed by the Section Editor Peng Zhang, MD, PhD (Department of Urology, Zhongnan Hospital of Wuhan University, Wuhan, China).

Conflicts of Interest: All authors have completed the ICMJE uniform disclosure form (available at http://dx.doi. org/10.21037/tcr.2019.05.27). The authors have no conflicts of interest to declare.

Ethical Statement: The authors are accountable for all 
aspects of the work in ensuring that questions related to the accuracy or integrity of any part of the work are appropriately investigated and resolved.

Open Access Statement: This is an Open Access article distributed in accordance with the Creative Commons Attribution-NonCommercial-NoDerivs 4.0 International License (CC BY-NC-ND 4.0), which permits the noncommercial replication and distribution of the article with the strict proviso that no changes or edits are made and the original work is properly cited (including links to both the formal publication through the relevant DOI and the license). See: https://creativecommons.org/licenses/by-nc-nd/4.0/.

\section{References}

1. Kyriakopoulos CE, Chen YH, Carducci MA, et al. Chemohormonal Therapy in Metastatic HormoneSensitive Prostate Cancer: Long-Term Survival Analysis of the Randomized Phase III E3805 CHAARTED Trial. J Clin Oncol 2018;36:1080-7.

2. Fizazi K, Tran N, Fein L, et al. Abiraterone plus Prednisone in Metastatic, Castration-Sensitive Prostate Cancer. N Engl J Med 2017;377:352-60.

3. Parker CC, James ND, Brawley CD, et al. Radiotherapy

Cite this article as: Harper B, Klaassen Z, Wallis CJD. Local therapy in patients with metastatic prostate cancer: a new standard of care? Transl Cancer Res 2019;8(Suppl 6):S592-S594. doi: $10.21037 /$ tcr.2019.05.27 to the primary tumour for newly diagnosed, metastatic prostate cancer (STAMPEDE): a randomised controlled phase 3 trial. Lancet 2018;392:2353-66.

4. Wallis CJD, Klaassen Z, Bhindi B, et al. Comparison of Abiraterone Acetate and Docetaxel with Androgen Deprivation Therapy in High-risk and Metastatic Hormone-naïve Prostate Cancer: A Systematic Review and Network Meta-analysis. Eur Urol 2018;73:834-44.

5. Bernard B, Gershman B, Karnes RJ, et al. Approach to Oligometastatic Prostate Cancer. Am Soc Clin Oncol Educ Book 2016;35:119-29.

6. Battaglia A, De Meerleer G, Tosco L, et al. Novel Insights into the Management of Oligometastatic Prostate Cancer: A Comprehensive Review. Eur Urol Oncol 2019;2:174-88.

7. Boevé LMS, Hulshof MCCM, Vis AN, et al. Effect on Survival of Androgen Deprivation Therapy Alone Compared to Androgen Deprivation Therapy Combined with Concurrent Radiation Therapy to the Prostate in Patients with Primary Bone Metastatic Prostate Cancer in a Prospective Randomised Clinical Trial: Data from the HORRAD Trial. Eur Urol 2019;75:410-8.

8. Burdett $\mathrm{S}$, Boevé LM, Ingleby FC, et al. Prostate Radiotherapy for Metastatic Hormone-sensitive Prostate Cancer: A STOPCAP Systematic Review and Metaanalysis. Eur Urol 2019;76:115-24. 